\title{
Trends in heart disease mortality among breast cancer survivors in the US, 1975-2017
}

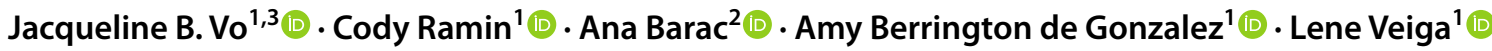

Received: 2 August 2021 / Accepted: 3 January 2022 / Published online: 2 February 2022

This is a U.S. government work and not under copyright protection in the U.S.; foreign copyright protection may apply 2022

\begin{abstract}
Purpose Heart disease is a significant concern among breast cancer survivors, in part due to cardiotoxic treatments including chemotherapy and radiotherapy. Long-term trends in heart disease mortality have not been well characterized. We examined heart disease mortality trends among US breast cancer survivors by treatment type.

Methods We included first primary invasive breast cancer survivors diagnosed between 1975 and 2016 (aged 18-84; survived $12+$ months; received initial chemotherapy, radiotherapy, or surgery) in the SEER-9 Database. Standardized mortality ratios (SMRs) and 10-year cumulative heart disease mortality estimates accounting for competing events were calculated by calendar year of diagnosis and initial treatment regimen. $P_{\text {trends }}$ were assessed using Poisson regression. All statistical tests were 2 -sided.

Results Of 516,916 breast cancer survivors, 40,812 died of heart disease through 2017. Heart disease SMRs declined overall from 1975-1979 to 2010-2016 (SMR 1.01 [95\%CI: 0.98, 1.03] to 0.74 [0.69, 0.79], $p_{\text {trend }}<0.001$ ). This decline was also observed for survivors treated with radiotherapy alone and chemotherapy plus radiotherapy. A sharper decline in heart disease SMRs was observed from 1975 to 1989 for left-sided radiotherapy, compared to right-sided. In contrast, there was a non-significant increasing trend in SMRs for chemotherapy alone, and significant by regional stage ( $p_{\text {trend }}=0.036$ ). Largest declines in 10-year cumulative mortality were observed from 1975-1984 to 2005-2016 among surgery only: 7.02\% (95\%CI: $6.80 \%, 7.23 \%)$ to $4.68 \%$ (95\%CI: $4.39 \%, 4.99 \%$ ) and radiotherapy alone: $6.35 \%$ (95\% CI: $5.95 \%, 6.77 \%$ ) to $2.94 \%$ (95\% CI: $2.73 \%, 3.16 \%$ ).

Conclusions We observed declining heart disease mortality trends by most treatment types yet increasing for regional stage patients treated with chemotherapy alone, highlighting a need for additional studies with detailed treatment data and cardiovascular management throughout cancer survivorship.
\end{abstract}

Keywords Breast cancer $\cdot$ Cancer survivorship $\cdot$ Heart disease mortality $\cdot$ Descriptive epidemiology

Jacqueline B. Vo

jacqueline.vo@nih.gov

1 Radiation Epidemiology Branch, Division of Cancer Epidemiology and Genetics, National Cancer Institute, Bethesda, MD, USA

2 Director of Cardio-Oncology and Professor of Medicine, Medstar Heart and Vascular Institute, Georgetown University, Washington, DC, USA

3 Present Address: Cancer Prevention Fellowship Program, Division of Cancer Prevention, Bethesda, MD, USA

\section{Background}

Advances in cancer treatment have contributed to the 5-year survival rates among breast cancer survivors approaching $90 \%$ [1]. These advances have shifted attention to the late adverse effects of treatment. Cardiovascular disease is now the leading cause of non-cancer deaths in women diagnosed with breast cancer in the US [2, 3]. Chemotherapy and radiotherapy both increase the risk of cardiovascular disease, and these adverse effects can occur acutely during treatment or decades after treatment has ended. Among older breast cancer survivors, the risk of dying from heart disease is now greater than breast cancer itself [2].

Etiology of cardiotoxicity varies by cancer treatment type and time since diagnosis. Anthracyclines and trastuzumab 
are associated with the risk of cardiomyopathy and heart failure, and the risk is significantly higher when used together [4-7]. Heart failure may present after anthracycline use, when ventricular dilation and dysfunction may be irreversible [7]. Limited data are available on the long-term cardiotoxic effects of trastuzumab; however, in the absence of anthracycline therapy, its cardiac effects are believed to be largely reversible after cessation of treatment [7]. Radiotherapy is associated with coronary artery disease and appears approximately 5-20 years after treatment completion [7-10].

Increased understanding of treatment-related adverse effects for breast cancer survivors has contributed to declines in anthracycline use [11], selection of alternative, less cardiotoxic treatments with similar effectiveness in certain breast cancers [12,13], as well as implementation of modern radiotherapy techniques with reduced radiation exposure to the heart [14]. These adaptations in cancer treatment and increased awareness of cardiotoxicity should have impacted heart disease mortality rates among breast cancer survivors. Yet, potential shifts in heart disease mortality among US breast cancer survivors by treatment type over time have not been well characterized. In this study, we examined the long-term trends in heart disease mortality among women diagnosed with a first primary invasive breast cancer in the US using data from the Surveillance, Epidemiology, and End Results (SEER)-9 cancer registries according to initial treatment regimen (chemotherapy, radiotherapy, or surgery) and breast cancer characteristics from 1975 to 2017.

\section{Methods}

\section{Data source}

This retrospective, population-based cohort study utilized the SEER-9 database for breast cancer survivors, and reference cohort from the US Mortality data. SEER-9 registries cover 9 registries throughout the USA and represent approximately $9.4 \%$ of the US population and leverages cancer registry data to characterize cancer incidence, treatment, and survival. US Mortality Data are maintained by the National Center for Health Statistics (NCHS) of the Centers for Disease Control and Prevention (CDC). Causes of death and population data were ascertained from death certificates. This research was exempt of Institutional Review Board by the National Institutes of Health Office of Human Subjects Research based on the usage of deidentified existing data.

\section{Study population}

We studied long-term patterns of heart disease mortality among 516,916 women diagnosed with a first primary, invasive breast cancer between ages 18-84, survived 12 months or longer, and received initial surgery, chemotherapy, or radiotherapy within SEER-9. We included first primary breast cancer only since treatment information in SEER is limited to first course of treatment. Women were diagnosed with breast cancer between January 1, 1975 and December 31, 2016 with follow-up until December 31, 2017.

\section{Heart disease mortality}

Our outcome of interest was heart disease mortality identified using consistent site groupings over time by the International Classification of Diseases (ICD)-8 (1976-1978) and ICD-9 (1979-1998): 390-398, 402, 404, 410-429; and ICD-10 codes (1999-2017): I00-I09, I11, I13, I20-I51. Heart disease mortality was ascertained since this is a clinically relevant outcome for the selected treatments and the breast cancer population $[7,15]$.

\section{Treatment information}

We classified women according to their initial breast cancer treatment: surgery only (women who received surgery but no/unknown history of chemotherapy or radiotherapy; $n=197,449$ ), chemotherapy alone (women who received chemotherapy, with or without surgery, but no/unknown history of radiotherapy; $n=76,479$ ), chemotherapy plus radiotherapy (women who received both chemotherapy and radiotherapy, with or without surgery; $n=102,838$ ), and radiotherapy alone (women who received radiotherapy, with or without surgery, but no/unknown history of chemotherapy; $n=140,150)$. The treatment groups were mutually exclusive. Further, we present a subgroup analysis for radiotherapy alone by laterality of the breast cancer (left-sided and right-sided).

In the SEER database, trastuzumab was included under chemotherapy until 2013 when it was classified under biologic therapy/immunotherapy [16]. The SEER database does not provide detailed information on specific drugs, and therefore, we were unable to distinguish trastuzumab from other biologic therapy/immunotherapy agents from 2013 onward. To remain consistent, we considered trastuzumab under the chemotherapy treatment group for the entire study period and conducted a sensitivity analysis by including the biologic therapy/immunotherapy category in the chemotherapy group for diagnoses after 2013.

\section{Statistical analyses}

Patients were followed beginning 1 year after their first primary breast cancer diagnosis (to estimate treatment completion) until date of last contact, death, or end up study period (December 31, 2017), whichever came first. To investigate 
heart disease risk relative to general population, we calculated standardized mortality ratios (SMRs) and corresponding 95\% confidence intervals (CIs) comparing risk of heart disease deaths from the breast cancer cohort to the US general female population. SMRs were calculated by dividing the observed number of heart disease deaths among breast cancer survivors by the expected number in the US female general population, adjusted for age at death (5-year groups), race (White/Black/other), and year of death. We examined trends in heart disease SMRs by calendar year of breast cancer diagnosis and treatment type (surgery only/chemotherapy alone/chemotherapy plus radiotherapy/radiotherapy alone), latency defined as time since breast cancer diagnosis (1-9 years/10-19 years/20 + years), age of breast cancer diagnosis (18-49 years/50-59 years/60-69 years/70-84 ye ars), and breast cancer stage (localized/regional/distant) (described further in Supplement A).

Tests for trend of SMRs by calendar year of breast cancer diagnosis (continuous) were assessed using Poisson regression model, with expected events as the offset. A sensitivity analysis was conducted to examine the SMRs by censoring follow-up at a second invasive cancer diagnosis, as development of a second cancer could increase risk of mortality. To assess the clinical burden of heart disease mortality, we calculated cumulative mortality (and corresponding 95\% CIs) from heart disease, taking into account the competing risk of death from other causes [17]. We examined 10-year cumulative mortality estimates by calendar year of breast cancer diagnosis, stratified by treatment type. SMRs were conducted using SEER*Stat version 8.3.8. Cumulative mortality (using the stcompet package) and $p_{\text {trend }}$ tests were conducted in Stata version 16 (StataCorp, College Station, TX). All tests were 2-sided with statistical significance set at $p<0.05$.

\section{Results}

\section{Descriptive characteristics}

From 1975 to 2016, there were 516,916 one-year breast cancer survivors with a median follow-up time was 9.59 years (interquartile range of 4.58-16.84). Of these survivors, $38.20 \%$ received surgery only; $14.80 \%$ received chemotherapy alone; $19.89 \%$ received chemotherapy plus radiotherapy; and $27.11 \%$ received radiotherapy alone as their initial treatment (Table 1). The majority of localized cancers received either surgery only $(44.08 \%)$ or radiotherapy alone (35.26\%); regional cancers mostly received surgery only (29.48\%), chemotherapy alone $(23.72 \%)$, or chemotherapy plus radiotherapy $(33.53 \%)$; and distant cancers mostly received chemotherapy alone $(34.14 \%)$ or chemotherapy plus radiotherapy $(35.18 \%)$. Survivors who received surgery only or radiotherapy alone were older at breast cancer diagnosis than those who received chemotherapy alone or chemotherapy plus radiotherapy. Treatment patterns were broadly similar by race. Use of surgery only declined over time from $1975-1979$ to $2010-2016$ (69.19\% to $21.46 \%)$, whereas chemotherapy plus radiotherapy use (4.07\% to $28.71 \%$ ) and radiotherapy alone (17.74\% to $34.19 \%)$ increased over time. Overall, there were 246,205 deaths, and 40,812 (16.58\%) died of heart disease. A large proportion of survivors who died of heart disease had received surgery only (50.35\%), followed by radiotherapy alone (27.91\%).

\section{Heart disease SMRs}

\section{By treatment type}

Overall, heart disease mortality in breast cancer survivors declined significantly relative to the general population by the calendar year of breast cancer diagnosis, from an SMR of 1.01 (95\%CI: $0.98,1.03)$ in $1975-1979$ to SMR of 0.74 (95\%CI: $0.69,0.79)$ in $2010-2016, p_{\text {trend }}<0.001$ (Table 2). This declining trend in SMRs was also observed for patients treated with chemotherapy plus radiotherapy and radiotherapy alone. Left-sided radiotherapy had a greater decline in the SMRs for heart disease in the earlier years, from 1.77 (95\%CI: $1.64,1.91)$ in $1975-1979$ to 0.93 (95\%CI: $0.87,0.99$ ) in 1985-1989, compared to right-sided (SMR 1.39 [95\%CI: $1.27,1.51]$ to SMR 0.84 [95\%CI: 0.78,0.90] for same time period), while the decline in SMRs between these two groups after 1990 was similar. In contrast, there was a non-significant increasing trend for heart disease mortality among survivors treated with chemotherapy alone, with an SMR of 0.88 (95\%CI: 0.77, 0.99) in 1975-1979 that increased to 1.01 (95\%CI: $0.81,1.25)$ in 2010-2016, $p_{\text {trend }}=0.11$.

The trends in heart disease SMRs for chemotherapy were similar in the sensitivity analysis with the inclusion of biologic modifiers to the chemotherapy category after 2013 (Supplemental Table A), but SMRs were slightly attenuated when censored at second cancers (Supplemental Table B).

\section{By latency}

The increasing heart disease SMRs among patients treated with chemotherapy alone and the decreasing SMRs among patients treated with radiotherapy alone and chemotherapy plus radiotherapy were also broadly similar by latency period $(<10,10-19,20+$ years since diagnosis). Trends in heart disease SMRs were significantly increasing for patients treated with surgery only at $10+$ years after diagnosis and increasing for chemotherapy alone at 10-19 years after diagnosis (Supplemental Table C). 


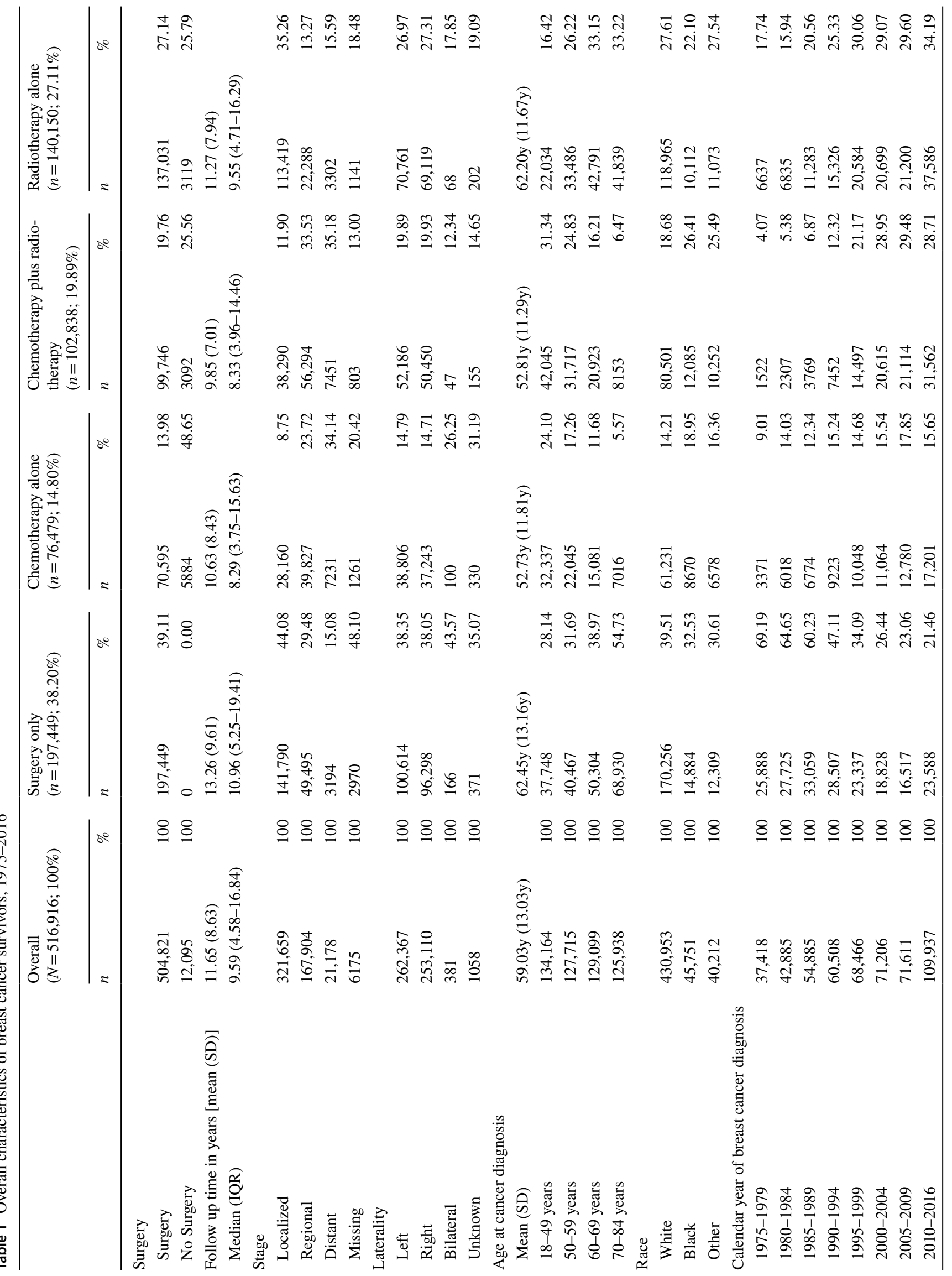




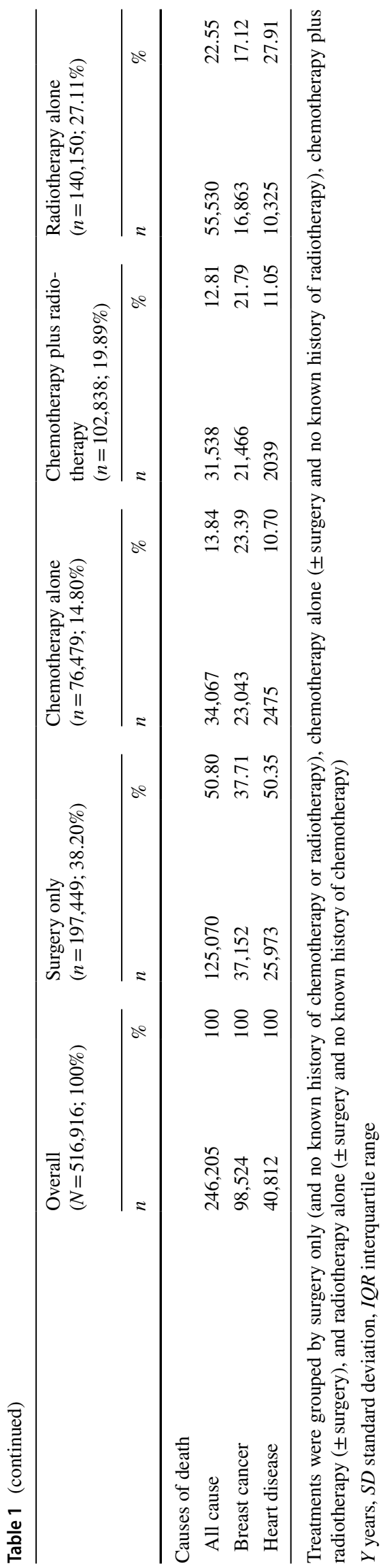

\section{By breast cancer stage}

Declining trends in heart disease SMRs were evident among all stages for radiotherapy alone and among localized and regional cancers for chemotherapy plus radiotherapy (Fig. 1). Conversely, trends for heart disease mortality significantly increased among women diagnosed with regional stage breast cancer who received chemotherapy alone with an SMR of 0.90 (95\%CI: 0.78, 1.04) from 1975-1979 to 1.00 (95\%CI: $0.70,1.37)$ in $2010-2016\left(p_{\text {trend }}=0.036\right)$.

\section{By age at breast cancer diagnosis}

There were significant declining trends in SMRs for heart disease among all age groups of patients that received chemotherapy plus radiotherapy and radiotherapy alone (Fig. 2; Supplemental Table D). The greatest decline was observed among the youngest survivors (18-49 years at diagnosis) for women treated with chemotherapy plus radiotherapy (SMR of 2.95 [95\%CI: 2.19, 3.89] from 1975-1979 to 1.11 [95\% CI: $0.59,1.89]$ in 2010-2016, $p_{\text {trend }}<0.001$ ) and for women treated with radiotherapy alone (SMR of 2.35 [95\%CI: 2.02, 2.72] declined to 0.68 [95\%CI: 0.19, 1.75] for the same time period, $p_{\text {trend }}<0.001$ ). For surgery only, there were increasing trends among women aged 18-59 and declining trends for older women.

\section{Cumulative mortality of heart disease}

Cumulative mortality analyses demonstrated the varying clinical burden of heart disease by calendar year of breast cancer diagnosis and treatment type (Fig. 3). The 10-year cumulative mortality declined among survivors diagnosed from 1975-1984 to 2005-2016 treated with surgery only: 7.02\% (95\% CI: $6.80 \%, 7.23 \%$ ) to $4.68 \%$ (95\% CI: $4.39 \%$, 4.99\%), and with radiotherapy alone: $6.35 \%$ (95\% CI: 5.95\%, $6.77 \%$ ) to $2.94 \%$ (95\% CI: $2.73 \%, 3.16 \%$ ). For the same time period, the declines in cumulative mortality of heart disease were smaller for the chemotherapy alone group: $2.38 \%$ (95\% CI: $2.09 \%, 2.70 \%$ ) to $1.58 \%$ (95\% CI: $1.38 \%, 1.79 \%$ ), and for the chemotherapy plus radiotherapy group: $1.78 \%$ (95\% CI: $1.40 \%, 2.24 \%$ ) to $1.21 \%$ (95\% CI: $1.08 \%, 1.79 \%$ ).

\section{Discussion}

To our knowledge, this is the first descriptive study to provide a novel perspective on trends in heart disease mortality among US breast cancer survivors, accounting for heart disease mortality rates in the general population, by treatment regimen over 42 years of follow-up. We observed a significant declining trend in heart disease mortality among breast cancer survivors compared to the general population 


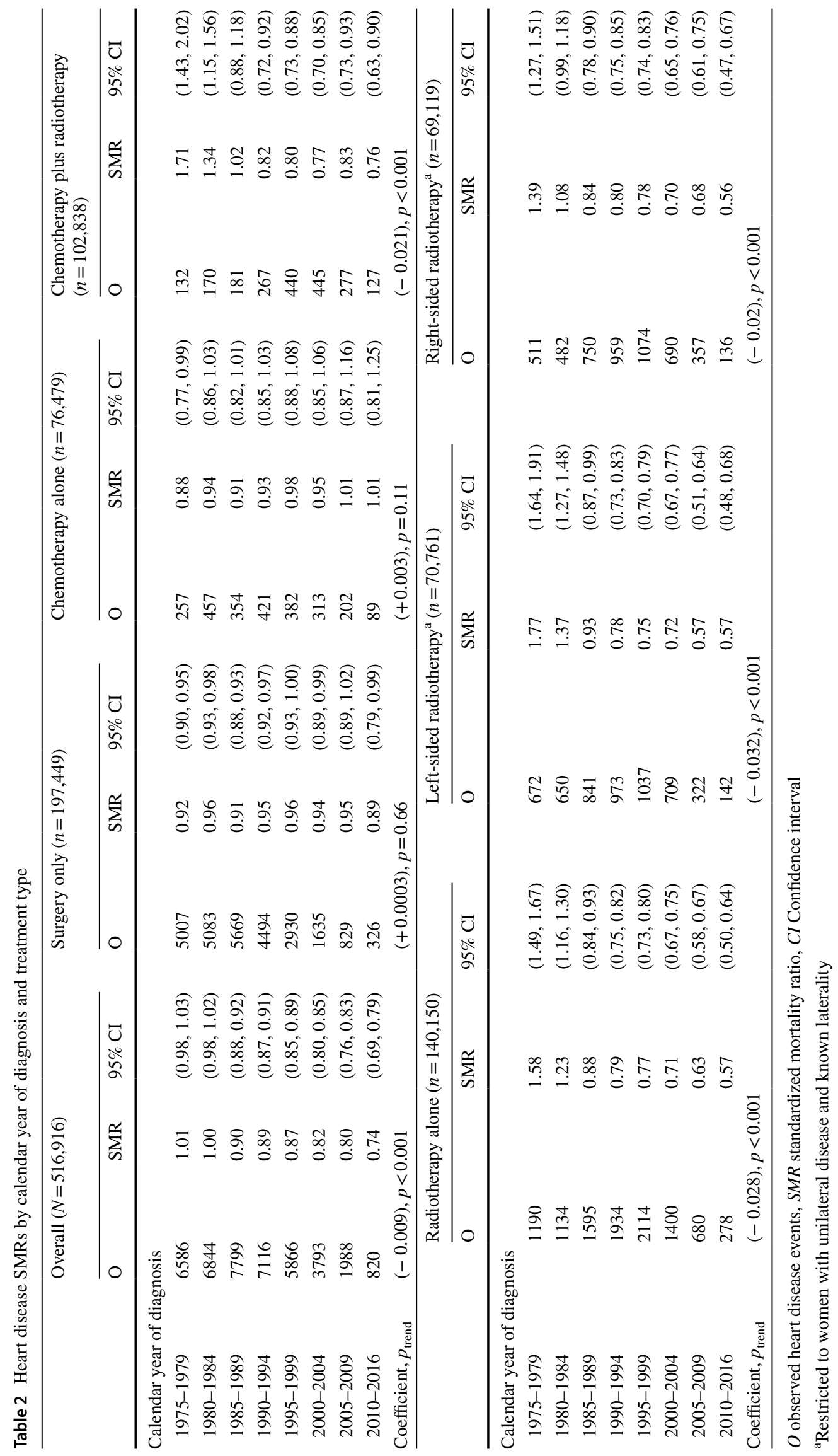



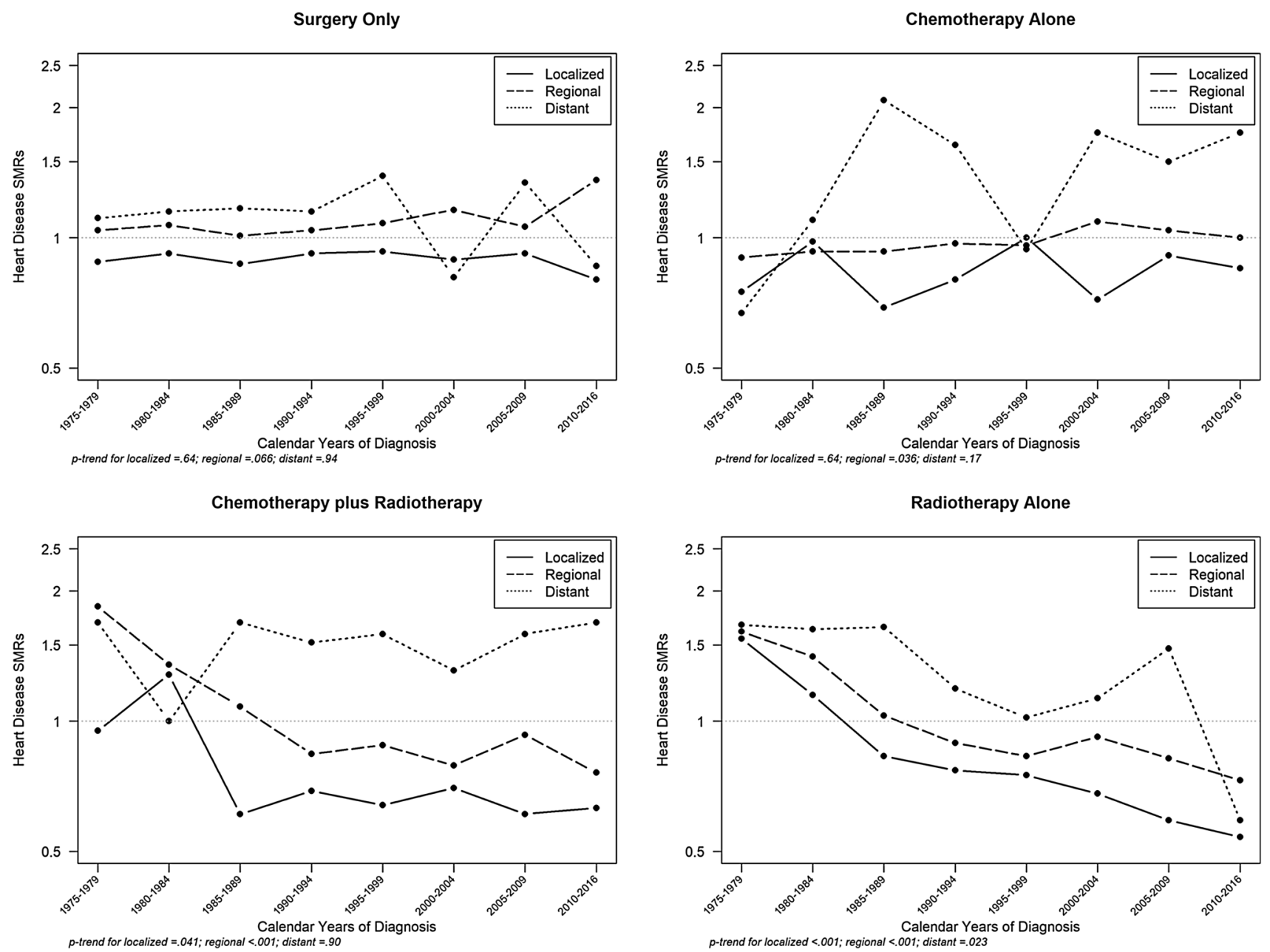

Fig. 1 Trends in heart disease SMRs among breast cancer survivors by stage at cancer diagnosis and treatment type. SMR standardized mortality ratios

by calendar year of diagnosis, overall and for breast cancer survivors treated with radiotherapy alone. The greatest declines were observed among younger survivors treated with radiotherapy. In contrast, we observed an increasing trend in heart disease mortality for regional stage patients treated with chemotherapy alone.

Several studies have reported increased risk of death due to heart disease among US breast cancer survivors [8, 18-21]. Yet, to date, only two studies have described patterns of heart disease mortality among breast cancer survivors compared to the general population, and these did not evaluate the trends according to treatment group $[22,23]$. Weberpals and colleagues studied patients treated more recently (2000-2011) and reported a lower risk of heart disease deaths among breast cancer survivors treated with chemotherapy or radiotherapy compared to the general population $(\mathrm{SMR}=0.84$ [95\% 0.79, 0.90]) [23]. Our study adds to the literature by describing the long-term trends which demonstrates the likely impact of treatment changes with higher SMRs in the early years, especially between 1975 and 1984 when heart disease mortality was elevated compared to the general population. Sturgeon et al. assessed overall cardiovascular disease (CVD) - and specific CVD disease mortality among 28 cancer types diagnosed between 1973 and 2012. Patients diagnosed with breast cancer were among six cancer sites with a larger proportion of CVD deaths which increased by calendar year of diagnosis, but the study did not examine SMRs by treatment group [22].

Overall, breast cancer survivors in the US had a lower risk of heart disease mortality compared to the general population. This lower risk in heart disease SMRs may partially be explained by the healthy screenee bias [24], where some patients have heightened medical surveillance after breast cancer diagnosis and better healthcare access, and the increasing awareness of cardiovascular risk factors among healthcare providers and patients. Additionally, breast cancer survivors may be more likely to die of breast cancer in 

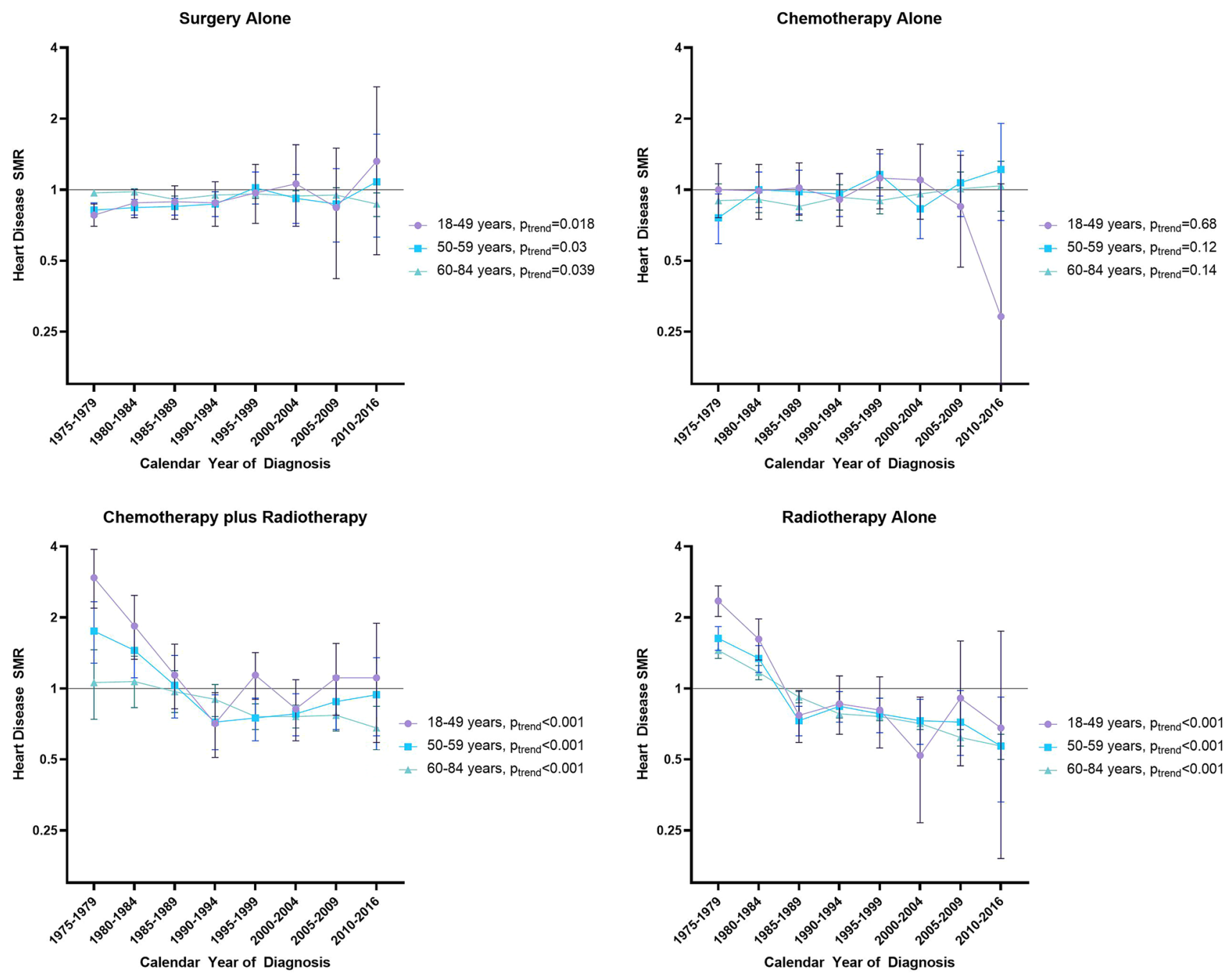

Fig. 2 Heart disease SMRs by calendar year of diagnosis, age at diagnosis, and treatment type. SMR standardized mortality ratios

the first several years after diagnosis [18, 19], which we then accounted for in the cumulative mortality analyses. By examining stratified SMRs for heart disease, our study revealed differences by treatment type and elevated heart disease mortality compared to the general population in selected time periods.

Our finding of a declining trend in heart disease mortality among breast cancer survivors treated with radiotherapy is consistent with the changes in treatment practice. With increasing awareness of cardiotoxicity over time, as evidenced with development of clinical guidelines [7, 15, 25], there have been advances in cardioprotection strategies to minimize potential heart damage including positioning patients to displace heart during radiotherapy administration, more precise radiotherapy using imaging and brachytherapy, and alternative radiotherapy options (i.e., proton therapy) with potentially lower heart disease risk [14]. Because of proximity to the heart, left breast radiotherapy has a higher risk of heart disease compared to the right breast [10]. We observed a greater declining trend among left breast radiotherapy in the early years when radiotherapy techniques delivered higher doses to the heart. Modern radiotherapy techniques to reduce exposure to the heart likely contributed to similar heart disease SMRs by laterality after 1990 . Results for the women treated with radiotherapy most recently should be interpreted cautiously because with shorter follow-up, cardiotoxicity may not yet be apparent.

The increase in heart disease mortality after chemotherapy alone among regional stage breast cancer survivors are likely driven by changing treatment patterns $[11,26]$, including increasing trastuzumab uptake. Trastuzumab received initial Federal Drug Administration (FDA) approval in 1998 and subsequent FDA approval for adjuvant treatment of HER2-positive node-positive breast cancers in 2006 [27]. HER2-positive breast cancers comprise of nearly $15 \%$ of breast cancer diagnoses [1], and the American Society of 

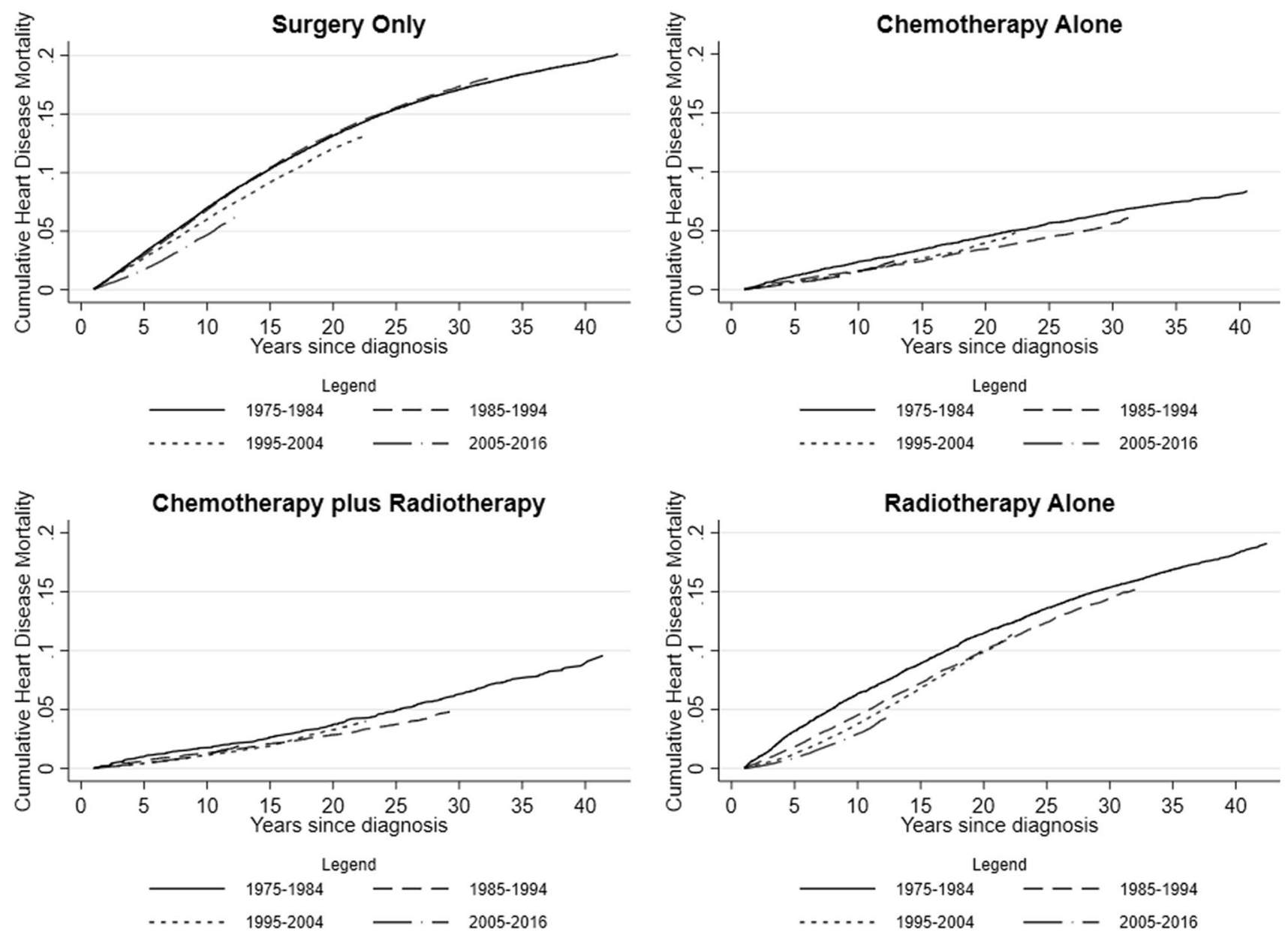

Fig. 3 Cumulative mortality for heart disease among breast cancer survivors, by treatment type and calendar year of diagnosis

Clinical Oncology recommends trastuzumab for all women with HER2-positive node-positive cancers [28, 29]. Trastuzumab is associated with declines in cardiac function (measured via left ventricular ejection fraction) and when used sequentially with anthracyclines, the risk of heart disease is exponentially greater $[6,15,20]$. Trastuzumab without anthracyclines has not demonstrated long-term increased heart disease risk in early breast cancer clinical trials [30]. While there is evidence of declining anthracycline use in breast cancer treatment [11], this relatively recent change is unlikely to have impacted patterns of heart disease mortality. Additionally, changing patterns in staging definitions, cancer treatment regimens, use of neoadjuvant chemotherapy, and inclusion of gene expression panels (e.g., Oncotype DX) to assess recurrence and guide treatment decisions [1] can affect trends in heart disease mortality. These variables are unavailable in the SEER database or for the entire study period, but we attempted to account for time period changes by stratifying by year of breast cancer diagnosis. Specifically, we estimated cumulative mortality by calendar period of diagnosis to account for changes in treatments. Future research into detailed cancer treatment data and associations with cardiotoxicity are warranted to explore these trends.

Our results demonstrated women treated with surgery only who were aged 18-59 at breast cancer diagnosis have an increasing trend in heart disease compared to the general population but declining for women aged 60-84 years. The etiology is unclear for contrasting heart disease trends for the surgery only group by age. The surgery only treatment group largely consisted of older women, and advancing age is a shared risk factor for both breast cancer and heart disease [7]. Potential under-reported treatment (e.g., chemotherapy or radiotherapy) among the surgery only group may misclassify some women, as women diagnosed with advanced cancers may likely receive additional treatment beyond surgery.

A strength of this study is the large size of the populationbased cohort and long-term follow-up. Our study has several limitations due to its registry-based design including lack of information on traditional cardiovascular disease risk factors (e.g., hypertension, diabetes, dyslipidemia), subsequent treatment, specific chemotherapy treatment agents, and potentially under-reported initial treatment. However, 
a previous report describe the sensitivity of chemotherapy and radiotherapy within SEER to be moderate $(69 \%$ and $80 \%$, respectively), and the positive predictive value high ( $91 \%$ and $98 \%$, respectively) for breast cancer [31]. Further, we were not able to evaluate SMRs by breast cancer subtype (estrogen receptor, progesterone receptor, and human epidermal growth factor receptor 2 status) or by hormone therapy use since these data were not available consistently throughout our follow-up.

Characterizing heart disease mortality among breast cancer survivors is an important step in the context of cardiooncology care and research. Breast cancer survivors are the largest group of cancer survivors, and maintaining optimal cardiovascular health is crucial as they are living many years post diagnosis and are at increased risk due to cancer treatment risk factors and mutual cancer and cardiovascular disease risk factors $[1,7,21]$. These mutual lifestyle and behavior risk factors include diet, obesity, physical activity, and smoking are associated with both the development of heart disease and breast cancer [7]. Independently, management of these risk factors can reduce breast cancer recurrence and prevent heart disease [32, 33]. While the SEER database lacks individual-level heart disease risk factors, the presented SMR analyses accounted for the declining heart disease mortality rates in the general population [34] reflecting changes in risk factors and treatment for heart disease. Studies and interventions to quantify and reduce risk factors for primordial heart disease prevention [33] among breast cancer survivors are an essential step.

Though we observed declining heart disease SMRs among breast cancer survivors treated with radiotherapy, it is important not to lose sight of this progress, as the association of heart disease still exists with radiotherapy use [8]. Clinical guidelines were developed for breast cancer survivors to recommend cardiovascular screening and assessment of cardiac function via cardiac magnetic resonance imaging (cMRI) or echocardiograms before treatment with anthracyclines, and before and during treatment when used sequentially with trastuzumab $[7,15,25,35]$. Yet guidelines only recently included recommendations for cardiac screening of high risk patients who received anthracyclines within 1 year of completion of treatment and do not have long-term guidelines for cardiac screening [15]. Studies have demonstrated late effects of cardiotoxicity from cancer treatment [18], in line with our increasing heart disease SMRs among regional stage breast cancer patients who received chemotherapy alone. Research detailing consequences of late occurring cardiotoxicity and the effects of screening on long-term reduction of heart disease risk are lacking.

In addition to screening recommendations, recent studies and current trials are examining the effectiveness of pharmacological cardiotoxicity prevention using angiotensinconverting enzyme (ACE) inhibitors, beta blockers, and statins, which can protect against declines in cardiac function [36, 37]. Their common use in the general population, combined with their potential for safe and effective use with known cardiotoxic treatment, may allow for cardiac protection while not compromising breast cancer survival. With the available data, clinical implications from this study further support inclusion of cardiovascular management and discussions of potential cardiotoxicity before, during, and throughout cancer survivorship. Clinical care should be individualized to the patient; however, it is essential to provide patient care concordant with clinical recommendations to ensure appropriate cardiovascular screenings are obtained (e.g., cMRI and echocardiograms) for breast cancer survivors receiving cardiotoxic cancer treatment.

In conclusion, we found that heart disease mortality among US breast cancer survivors has declined compared to the general population, overall and among patients treated with radiotherapy, but has increased among regional stage patients treated with chemotherapy. Further work is needed to understand specific chemotherapy agents that contribute to heart disease and to reduce the heart disease burden among breast cancer survivors.

Supplementary Information The online version contains supplementary material available at https://doi.org/10.1007/s10549-022-06515-5.

Author contributions JBV, $\mathrm{CR}, \mathrm{ABdG}$, and $\mathrm{LV}$ contributed to the conceptualization and design of the analysis. $\mathrm{AB}$ provided content expertise and feedback. JBV and LV led the investigation and statistical analysis with input from all authors. JBV and LV were responsible for the original draft of the manuscript, and all authors $(\mathrm{CR}, \mathrm{AB}$, $\mathrm{ABdG}$ ) made significant contributions to revising and finalizing the manuscript.

Funding This work was supported by the intramural research program of the National Cancer Institute at the National Institutes of Health.

Data availability The datasets analyzed during the current study are available from the SEER registry https://seer.cancer.gov/.

Code availability Available upon request.

\section{Declarations}

Conflict of interest The authors declare that they have no conflicts of interest.

Disclaimer The funder had no role in the design of the study; the collection, analysis, or interpretation of the data; the writing of the manuscript; or the decision to submit the manuscript for publication. The results reported here and the conclusions derived are the sole responsibility of the authors. The opinions expressed by the authors are their own and this material should not be interpreted as representing the official viewpoint of the U.S. Department of Health and Human Services, the National Institutes of Health or the National Cancer Institute.

Open Access This article is licensed under a Creative Commons Attribution 4.0 International License, which permits use, sharing, 
adaptation, distribution and reproduction in any medium or format, as long as you give appropriate credit to the original author(s) and the source, provide a link to the Creative Commons licence, and indicate if changes were made. The images or other third party material in this article are included in the article's Creative Commons licence, unless indicated otherwise in a credit line to the material. If material is not included in the article's Creative Commons licence and your intended use is not permitted by statutory regulation or exceeds the permitted use, you will need to obtain permission directly from the copyright holder. To view a copy of this licence, visit http://creativecommons. org/licenses/by/4.0/.

\section{References}

1. American Cancer Society (2019) Breast cancer facts \& figures 2019-2020. American Cancer Society Inc, Atlanta

2. Patnaik JL, Byers T, Diguiseppi C, Dabelea D, Denberg TD (2011) Cardiovascular disease competes with breast cancer as the leading cause of death for older females diagnosed with breast cancer: a retrospective cohort study. Breast Cancer Res Treat. https://doi.org/10.1186/bcr2901

3. Du XL, Fox EE, Lai D (2008) Competing causes of death for women with breast cancer and change over time from 1975 to 2003. Am J Clin Oncol 31(2):105-116. https://doi.org/10.1097/COC.0b013 e318142c865

4. Kenigsberg B, Wellstein A, Barac A (2018) Left ventricular dysfunction in cancer treatment. JACC: Heart Failure 6(2):87-95. https://doi.org/10.1016/j.jchf.2017.08.024

5. Barish R, Gates E, Barac A (2019) Trastuzumab-induced cardiomyopathy. Cardiol Clin 37(4):407-418. https://doi.org/10.1016/j. ccl.2019.07.005

6. Thavendiranathan P et al (2016) Breast cancer therapy-related cardiac dysfunction in adult women treated in routine clinical practice: a population-based cohort study. JCO 34(19):2239-2246. https:// doi.org/10.1200/JCO.2015.65.1505

7. Mehta LS et al (2018) Cardiovascular disease and breast cancer: where these entities intersect: a scientific statement from the American Heart Association. Circulation 137:30-66. https://doi.org/10. 1161/CIR.0000000000000556

8. Darby SC et al (2013) Risk of ischemic heart disease in women after radiotherapy for breast cancer. N Engl J Med 368(11):987-998. https://doi.org/10.1056/NEJMoa1209825

9. Curigliano $\mathrm{G}$ et al (2012) Cardiovascular toxicity induced by chemotherapy, targeted agents and radiotherapy: ESMO Clinical Practice Guidelines. Annals of Oncology 23(suppl 7):vii155-vii166. https:// doi.org/10.1093/annonc/mds293

10. Darby SC, McGale P, Taylor CW, Peto R (2005) Long-term mortality from heart disease and lung cancer after radiotherapy for early breast cancer: prospective cohort study of about 300000 women in US SEER cancer registries. Lancet Oncol 6(8):557-565. https://doi. org/10.1016/S1470-2045(05)70251-5

11. Giordano SH, Lin Y-L, Kuo YF, Hortobagyi GN, Goodwin JS (2012) Decline in the use of anthracyclines for breast cancer. J Clin Oncol 30(18):2232-2239. https://doi.org/10.1200/JCO.2011.40. 1273

12. Nitz U et al (2019) West German study PlanB trial: adjuvant four cycles of epirubicin and cyclophosphamide plus docetaxel versus six cycles of docetaxel and cyclophosphamide in HER2-negative early breast cancer. JCO 37(10):799-808. https://doi.org/10.1200/ JCO. 18.00028

13. Blum JL et al (2017) Anthracyclines in early breast cancer: the ABC trials-USOR 06-090, NSABP B-46-I/USOR 07132, and NSABP
B-49 (NRG Oncology). JCO 35(23):2647-2655. https://doi.org/10. 1200/JCO.2016.71.4147

14. Lenihan DJ, Cuculich P (2018) Cardioprotection during therapeutic radiation treatment: peeling the onion of radiation cardiotoxicity? Circ Heart Fail. https://doi.org/10.1161/CIRCHEARTFAILURE. 118.005294

15. Armenian SH et al (2017) Prevention and monitoring of cardiac dysfunction in survivors of adult cancers: American Society of Clinical Oncology Clinical Practice Guideline. JCO 35(8):893-911. https:// doi.org/10.1200/JCO.2016.70.5400

16. National Cancer Institute, "Trastuzumab," National Cancer Institute. Available at https://seer.cancer.gov/seertools/seerrx/rx/53c44af710 2c1290262dc11a/

17. Coviello V, Boggess M (2004) Cumulative incidence estimation in the presence of competing risks. Stand Genomic Sci 4(2):103-112. https://doi.org/10.1177/1536867X0400400201

18. Bradshaw PT, Stevens J, Khankari N, Teitelbaum SL, Neugut AI, Gammon MD (2016) Cardiovascular disease mortality among breast cancer survivors. Epidemiology 27(1):6-13. https://doi.org/10.1097/ EDE.0000000000000394

19. Ramin C et al (2021) All-cause and cardiovascular disease mortality among breast cancer survivors in CLUE II, a long-standing community-based cohort. JNCI: J Natl Cancer Inst 113(2):137-145. https://doi.org/10.1093/jnci/djaa096

20. Bowles EJA et al (2012) Risk of heart failure in breast cancer patients after anthracycline and trastuzumab treatment: a retrospective cohort study. J Natl Cancer Inst 104(17):1293-1305. https://doi. org/10.1093/jnci/djs317

21. Armenian SH et al (2016) Cardiovascular disease among survivors of adult-onset cancer: a community-based retrospective cohort study. JCO 34(10):1122-1130. https://doi.org/10.1200/JCO.2015.64.0409

22. Sturgeon KM et al (2019) A population-based study of cardiovascular disease mortality risk in US cancer patients. Eur Heart J. https:// doi.org/10.1093/eurheartj/ehz766

23. Weberpals J, Jansen L, Müller OJ, Brenner H (2018) Long-term heart-specific mortality among 347476 breast cancer patients treated with radiotherapy or chemotherapy: a registry-based cohort study. Eur Heart J 39(43):3896-3903. https://doi.org/10.1093/eurhe artj/ehy 167

24. Weiss NS, Rossing MA (1996) Healthy screenee bias in epidemiologic studies of cancer incidence. Epidemiology 7(3):5

25. Curigliano G et al (2020) Management of cardiac disease in cancer patients throughout oncological treatment: ESMO consensus recommendations. Ann Oncol 31(2):171-190. https://doi.org/10.1016/j. annonc.2019.10.023

26. Morton LM et al (2019) Association of chemotherapy for solid tumors with development of therapy-related myelodysplastic syndrome or acute myeloid leukemia in the modern era. JAMA Oncol 5(3):318. https://doi.org/10.1001/jamaoncol.2018.5625

27. Federal Drug Administration (2010) HERCEPTIN (trastuzumab)

28. Denduluri N et al (2016) Selection of optimal adjuvant chemotherapy regimens for human epidermal growth factor receptor 2 (HER2) - negative and adjuvant targeted therapy for HER2-positive breast cancers: an American Society of Clinical Oncology Guideline adaptation of the cancer care Ontario clinical practice guideline. JCO 34(20):2416-2427. https://doi.org/10.1200/JCO.2016.67.0182

29. Denduluri N et al (2018) Selection of optimal adjuvant chemotherapy and targeted therapy for early breast cancer: ASCO clinical practice guideline focused update. JCO 36(23):2433-2443. https:// doi.org/10.1200/JCO.2018.78.8604

30. Dent SF, Morse A, Burnette S, Guha A, Moore H (2021) Cardiovascular toxicity of novel HER2-targeted therapies in the treatment of breast cancer. Curr Oncol Rep 23(11):128. https://doi.org/10.1007/ s11912-021-01114-x 
31. Noone A-M et al (2016) Comparison of SEER treatment data with medicare claims. Med Care 54(9):e55-e64. https://doi.org/10.1097/ MLR.0000000000000073

32. Haykowsky MJ, Scott JM, Hudson K, Denduluri N (2017) Lifestyle interventions to improve cardiorespiratory fitness and reduce breast cancer recurrence. American Society of Clinical Oncology Educational Book, Alexandria

33. Chomistek AK, Chiuve SE, Eliassen AH, Mukamal KJ, Willett WC, Rimm EB (2015) Healthy lifestyle in the primordial prevention of cardiovascular disease among young women. J Am Coll Cardiol 65(1):43-51. https://doi.org/10.1016/j.jacc.2014.10.024

34. Virani SS et al (2021) Heart disease and stroke statistics-2021 update: a report from the American Heart Association. Circulation. https://doi.org/10.1161/CIR.0000000000000950

35. National Comprehensive Cancer Network (2020) NCCN clinical practice guidelines in oncology (NCCN Guidelines: Survivorship v2.20)
36. Pituskin E et al (2017) Multidisciplinary approach to novel therapies in cardio-oncology research (MANTICORE 101-Breast): a randomized trial for the prevention of trastuzumab-associated cardiotoxicity. JCO 35(8):870-877. https://doi.org/10.1200/JCO.2016.68. 7830

37. Obasi M et al (2021) Statins to mitigate cardiotoxicity in cancer patients treated with anthracyclines and/or trastuzumab: a systematic review and meta-analysis. Cancer Causes Control. https://doi.org/ 10.1007/s10552-021-01487-1

Publisher's Note Springer Nature remains neutral with regard to jurisdictional claims in published maps and institutional affiliations. 